University of New Hampshire

University of New Hampshire Scholars' Repository

Space Science Center

Institute for the Study of Earth, Oceans, and

Space (EOS)

8-1999

\title{
Development of a Hard X-Ray Polarimeter for Astrophysics
}

\author{
Mark L. McConnell \\ University of New Hampshire - Main Campus, mark.mcconnell@unh.edu \\ John R. Macri \\ University of New Hampshire - Main Campus, John.Macri@unh.edu \\ M McClish \\ University of New Hampshire - Main Campus \\ James M. Ryan \\ University of New Hampshire, James.Ryan@unh.edu \\ D J. Forrest \\ University of New Hampshire - Main Campus
}

See next page for additional authors

Follow this and additional works at: https://scholars.unh.edu/ssc

Part of the Astrophysics and Astronomy Commons

\section{Recommended Citation}

McConnell, M.L.; Macri, J.R.; McClish, M.; Ryan, J.; Forrest, D.J.; Vestrand, W.T., "Development of a hard Xray polarimeter for astrophysics," Nuclear Science, IEEE Transactions on , vol.46, no.4, pp.890,896, Aug 1999 doi: $10.1109 / 23.790699$

This Conference Proceeding is brought to you for free and open access by the Institute for the Study of Earth, Oceans, and Space (EOS) at University of New Hampshire Scholars' Repository. It has been accepted for inclusion in Space Science Center by an authorized administrator of University of New Hampshire Scholars' Repository. For more information, please contact Scholarly.Communication@unh.edu. 


\section{Authors}

Mark L. McConnell, John R. Macri, M McClish, James M. Ryan, D J. Forrest, and W T. Vestrand 


\title{
Development of a Hard X-Ray Polarimeter for Astrophysics
}

\author{
M.L. McConnell, J.R. Macri, M. McClish, J. Ryan, D.J. Forrest and W.T. Vestrand \\ Space Science Center, Morse Hall, University of New Hampshire, Durham, New Hampshire 03824
}

\begin{abstract}
We have been developing a Compton scatter polarimeter for measuring the linear polarization of hard X-rays (100-300 $\mathrm{keV}$ ) from astrophysical sources. A laboratory prototype polarimeter has been used to successfully demonstrate the reliability of our Monte Carlo simulation code and to demonstrate our ability to generate a polarized photon source in the lab. Our design concept places a self-contained polarimeter module on the front-end of a a 5-inch positionsensitive PMT (PSPMT). We are currently working on the fabrication of a science model based on this PSPMT concept. Although the emphasis of our development effort is towards measuring hard X-rays from solar flares, our design has the advantage that it is sensitive over a rather large field-of-view ( $>$ 1 steradian), a feature that makes it especially attractive for $\gamma$-ray burst studies.
\end{abstract}

\section{INTRODUCTION}

The basic physical process used to measure linear polarization of hard X-rays $(100-300 \mathrm{keV})$ is Compton scattering [1]. The scattering geometry can be described by two angles. The first of these is the Compton scatter angle $(\theta)$,the angle between the incident and scattered photons. A second angle $(\eta)$ defines the scattered photon direction as projected onto a plane perpendicular to the incident photon direction. This angle, which we refer to as the azimuthal scatter angle, is measured from the plane containing the electric vector of the incident photon. For a given value of $\theta$, the scattering cross section for polarized radiation reaches a minimum at $\eta=0^{\circ}$ and a maximum at $\eta=90^{\circ}$. In other words, photons tend to be scattered at right angles relative to the plane of polarization of the incident radiation. In the case of a Compton scatter polarimeter, this asymmetry, which is maximized for values of $\theta$ near $90^{\circ}$, is exploited as a means to determine the linear polarization parameters of the incident radiation.

The successful design of a polarimeter hinges on the ability to reconstruct the kinematics of each event. In this context, we can consider: 1) the ability to measure the energies of both the scattered photon and the scattered electron; and 2) the ability to measure the scattering geometry.

A Compton scatter polarimeter consists of two detectors that are used to measure the energies of both the scattered photon and the scattered electron [2,3]. These measurements also serve to define the scattering geometry. One detector (the scattering detector) provides the medium for the Compton interaction to take place. This detector must be designed to maximize the probability of a single Compton interaction with a subsequent escape of the scattered photon. This implies a low-Z material that is sufficiently thick to induce a single Compton scattering, but thin enough to minimize the chance of subsequent interactions. The second detector (the calorimeter) absorbs the remaining energy of the scattered photon. Information regarding the scattering geometry comes from the relative location of the detectors. The accuracy with which the scattering geometry can be measured determines the ability to define the modulation pattern and therefore has a direct impact on the polarization sensitivity.

With regard to the definition of the modulation pattern (which follows a $\cos 2 \eta$ distribution), it is customary to define, as a figure-of-merit for the polarimeter, the polarization modulation factor [2,3]. For a given energy and incidence angle for an incoming photon beam, this can be expressed as,

$$
\mu_{P}=\frac{C_{\max }(P)-C_{\min }(P)}{C_{\max }(P)+C_{\min }(P)}
$$

where $C_{\max }$ and $C_{\min }$ are the maximum and minimum number of counts registered in the polarimeter, respectively, with respect to the azimuthal scatter angle $(\eta)$. It is useful to define the modulation factor which results from an incident beam that is $100 \%$ polarized,

$$
\mu_{100}=\frac{C_{\max }(100 \%)-C_{\min }(100 \%)}{C_{\max }(100 \%)+C_{\min }(100 \%)}
$$

We then use this result, together with the observed modulation factor $\left(\mu_{\mathrm{p}}\right)$, to determine the level of polarization in a measured beam,

$$
P=\frac{\mu_{P}}{\mu_{100}}=\frac{1}{\mu_{100}} \frac{C_{\max }(P)-C_{\min }(P)}{C_{\max }(P)+C_{\min }(P)}
$$

The $3 \sigma$ sensitivity for measuring polarization is then [2],

$$
P(3 \sigma)=\frac{3}{\mu_{100} s}\left[\frac{2(S+B)}{T}\right]^{1 / 2}
$$

where $S$ is the source count rate, $B$ is the background count rate, $\mu_{100}$ is the modulation factor for $100 \%$ polarization and $T$ is the observation time. We see that improved sensitivity to source polarization can be achieved either by increasing the modulation factor $\left(\mu_{100}\right)$ or by increasing the effective area of the polarimeter (thereby increasing the source count rate).

\section{LABORATORY PROTOTYPE}

In an earlier paper, we discussed a polarimeter design consisting of a ring of twelve individual scattering detectors (composed of low-Z plastic scintillator) surrounding a single Nal calorimeter [4]. To be recorded as a polarimeter event, an incident photon Compton scatters from one (and only one) of the scattering detectors into the central calorimeter. The incident photon energy can be determined from the sum of the energy losses in both detectors and the azimuthal scattering angle $(\eta)$ can be determined by the azimuthal angle of the associated scattering detector. When the polarimeter is arranged 
so that the incident flux is parallel to the symmetry axis, unpolarized radiation will produce an axially symmetric coincidence rate. If, on the other hand, the incident radiation is linearly polarized, then the coincidence rate will show an azimuthal asymmetry whose phase depends on the position angle of the incident radiation's electric field vector and whose magnitude depends on the degree of polarization. The characteristics of this design were investigated using a series of Monte Carlo simulations that were based on a modified version of the GEANT simulation package.

A prototype of this design was tested in the laboratory, in part to validate our Monte Carlo code [5,6]. For testing purposes, we set up a semicircular array of plastic scintillator elements around a central NaI detector. This semicircular design retained the fundamental physics, but, by eliminating the redundancy, simplified the hardware and associated electronics. A photograph of the laboratory setup is shown in Figure 1.

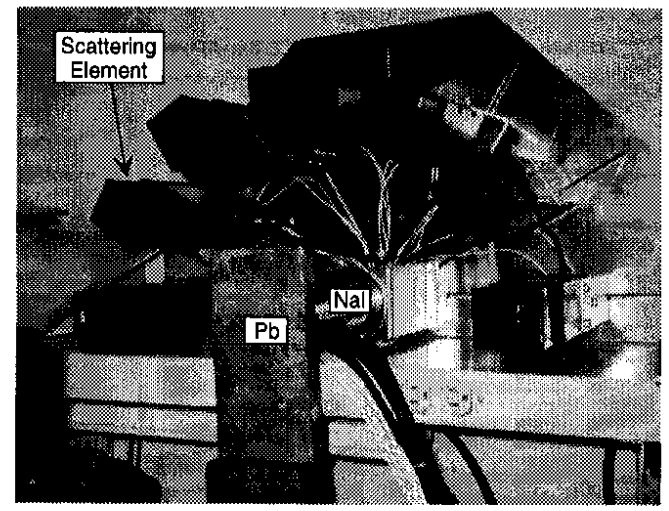

Figure 1: The laboratory prototype showing the plastic scattering elements surrounding the central NaI detector. The lead block was used to shield the NaI detector from direct flux.

A source of polarized photons was generated by Compton scattering photons from a radioactive source [7]. The exact level of polarization is dependent on both the initial photon energy and the photon scatter angle [6,8]. The use of plastic scintillator as a scattering block in generating the polarized beam permits the electronic tagging of the scattered (polarized) photons. This is especially useful in identifying (via coincidence techniques) the interaction of the polarized photons in the polarimeter.

Results from the prototype testing are shown in Figures 2 . and 3, where we show the measured data along with Monte Carlo simulation results for two different polarization angles. The polarization values derived from these data agree well with that expected from the laboratory polarization geometry. These results demonstrated: a) the ability of a simple Compton scatter polarimeter to measure hard X-ray polarization; b) the ability of our Monte Carlo code to predict the polarimeter response; and c) the ability to generate a source of polarized photons using a simple scattering technique.

\section{DESIGNING A HARD X-RAY POLARIMETER}

The goal of our program has been to develop a hard X-ray polarimeter that would be suitable for studying solar flare

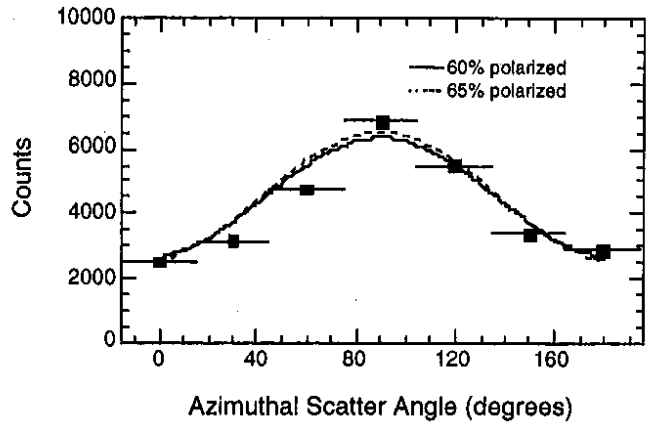

Figure 2: The prototype response to a polarized beam incident on-axis. The smooth curves represent simulation results.

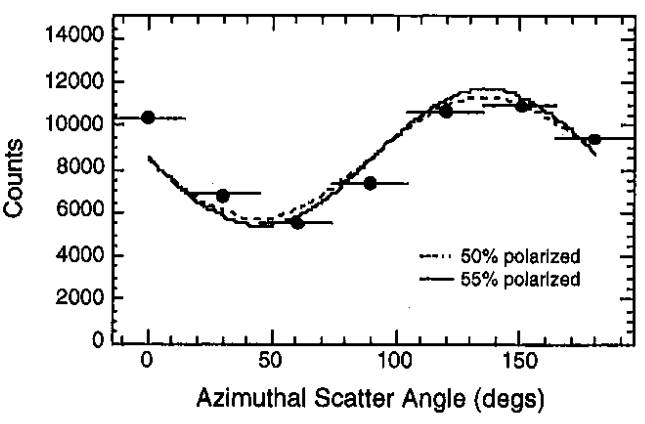

Figure 3: The prototype response to a polarized beam incident on-axis, but with a polarization angle rotated $\sim 45^{\circ}$ with respect to that in Figure 4 . The smooth curves represent simulation results.

emissions during the upcoming solar maximum. Such a polarimeter must meet the following requirements: 1) it must be compact and light-weight in order to conform with various budget restrictions imposed on any realistic payload; 2 ) it must be modular in order to provide flexibility as a piggy-back payload and to permit building up an array of detectors with sufficient sensitivity; 3) it must have reasonable detection efficiency over a broad energy range (100-300 keV); and 4) it must have polarization sensitivity below $10 \%$ in the $100-300$ $\mathrm{keV}$ energy range for a moderately-sized (class M5) solar flare. (Based on SMM-GRS observations during the 1980-82 solar maximum, we can expect $>50$ flares of class M5 or larger during the upcoming solar maximum period.)

\section{A. Design Considerations}

There are at least two possible means of improving the polarimeter performance over that of the laboratory prototype: 1) by more precisely measuring the scattering geometry of each event; and 2) by rejecting those events that undergo multiple Compton scattering within the scattering elements. A better geometry definition will serve to more clearly define the modulation pattern of the incident flux. Improved rejection of multiple scatter events will reduce the contribution of such events to the unmodulated component of the polarization response. Our simulations indicate that roughly $30-40 \%$ of the events recorded in the prototype polarimeter as valid events involved multiple scattering within a single scatter element. 
An improvement in the measured scattering geometry of an event can be achieved by improving the spatial resolution within each detector element. Fully 3-dimensional spatial information is generally not crucial. Since we are principally interested in the azimuthal scattering angle $(\mu)$ of each event, spatial information in the $x-y$ plane (i.e., parallel to the front surface of the polarimeter) will be of greatest importance. Although dependent on the precise geometry of the polarimeter, additional information regarding the $\mathrm{z}$-component of the location will generally add little to the information content of the event:

At these energies (100-300 keV), multiple scatter events in the central calorimeter can be safely ignored due to the dominance of the photoelectric effect (assuming that the calorimeter consists of some high-Z inorganic scintillator such as $\mathrm{NaI}$ or $\mathrm{CsI}$. Multiple scatter events can be important when the pathlength through the scattering elements becomes comparable to the mean free path of the incident photons (about $6 \mathrm{~cm}$ at $100 \mathrm{keV}$ ). Since the detection efficiency is, to a great extent, proportional to volume, the geometry of the scattering elements (in terms of both surface area and depth) must be carefully chosen so as to reach a compromise between detection efficiency and the generation of multiple scatter events. If, on the other hand, one can acquire information about the spatial distribution of energy deposits, it then becomes possible to distinguish those events with more than one interaction site (i.e., multiple scatter events). Such events can subsequently be rejected during the analysis. This capability would permit the effective use of larger volumes of plastic scintillator, with the potential for a subsequent increase in polarimeter sensitivity. Given the relatively large mean free path of the photons at these energies, a spatial resolution of $\sim 1.0 \mathrm{~cm}$ is sufficient to reject a large fraction of the multiple scatter events. Smaller spatial resolutions may be desirable for improving the definition of the scatter geometry.

Two other practical considerations should be noted. In order to reduce accidental coincidences that may be associated with high incident flux levels (such as that from a solar flare), there is a need to shield the calorimeter detectors from direct flux. A thin layer of lead ( $5 \mathrm{~mm}$ thick) is sufficient for this purpose. A second consideration is that of systematic variations in the azimuthal scatter angle distribution due, for example, to detection nonuniformities in the scattering elements. One way to ameliorate this condition is by continuously rotating the polarimeter about its axis of symmetry.

\section{B. A Baseline Polarimeter Design}

Based on the above considerations, we have developed a new conceptual design that places an entire device on the front end of a single 5-inch diameter position-sensitive PMT (PSPMT) [6]. Since the focus of our efforts have so far been directed toward solar studies, we refer to this new design as SOLPOL (for SOLar POLarimeter). The design incorporates a array of plastic scintillator elements to provide the improved spatial resolution in the scattering medium and to improve the rejection of multiple scatter events. The plastic elements are arranged in the form of an annulus having an outside diameter of $10 \mathrm{~cm}$ (corresponding to the sensitive area of the Hammatsu R3292 5-inch PSPMT). The central portion of the annulus is large enough to insert a small $2 \times 2$ array of $1 \mathrm{~cm} \mathrm{CsI}$



Figure 4: The SOLPOL polarimeter design showing the layout of the plastic scintillator elements and CsI elements on the front surface of a PSPMT. As shown here, the depth of the detector elements is $5.08 \mathrm{~cm}$.

scintillators. The CsI scintillators would be coupled to their own read-out devices for the energy measurement and signal timing.

Based on this concept, we have defined the baseline polarimeter design depicted in Figure 4. The scattering medium consists of an array of $5 \mathrm{~mm} \times 5 \mathrm{~mm}$ scintillator rods, each with a length of $5.08 \mathrm{~cm}$. The calorimeter medium consists of an array of $1 \mathrm{~cm} \times 1 \mathrm{~cm}$ CsI scintillators, each of which also has a length of $5.08 \mathrm{~cm}$. An ideal SOLPOL event is one in which the incident photon Compton scatters in one plastic element, with the remaining photon energy subsequently absorbed in the central CsI array.

We have completed a series of Monte Carlo simulations to determine the charcteristics of this baseline design. These simulations assume that we are able to uniquely identify which plastic scintillator element is involved in the event. The small cross-sectional area of each scintillator element ensures that practically all multiple scatter events are rejected. The energy threshold levels, particularly in the scattering elements, have a significant influence on the performance of the polarimeter at low energies. For the simulations, we have assumed a 

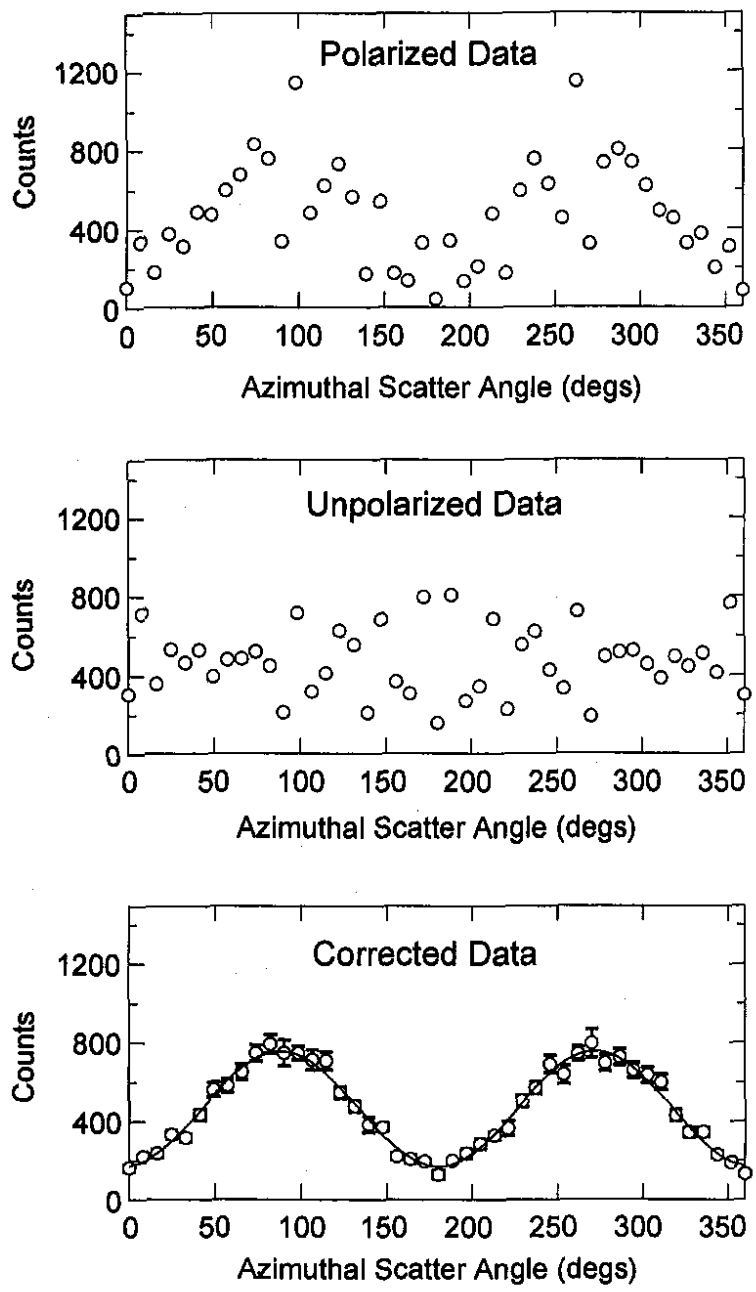

Figure 5: Simulated polarimeter data showing how the measured data is corrected for intrinsic geometric effects to extract the true modulation pattern. These data correspond to the response of the baseline SOLPOL design to a monoenergetic beam of $150 \mathrm{keV}$ photons incident at $0^{\circ}$.

threshold energy of $15 \mathrm{keV}$ in both the plastic and CsI scintillators.

Figure 5 illustrates the nature of the SOLPOL data. In this case, the data are from Monte Carlo simulations using the baseline SOLPOL design (Figure 4). The first panel shows the polarization response to a fully polarized monoenergetic beam of $150 \mathrm{keV}$ photons vertically incident on the front surface of the polarimeter. This distribution includes not only the intrinsic modulation pattern due to the Compton scattering process, but it also includes geometric effects related to the specific layout of the detector elements within the polarimeter and the associated quantization of possible scatter angles. The geometric effects can be more clearly seen in the case of an incident beam that is completely unpolarized, as shown in the second panel of Figure 5. (In practice, for analyzing real data, this unpolarized distribution would be determined by smulations rather than by direct measurements.) To extract the true distribution of polarized events, we divide the polarized distribution by the unpolarized distribution and normalize by the average of the unpolarized distribution. Only when we correct the raw data in this fashion do we clearly see the $\cos 2 \eta$ modulation pattern that is expected (the third panel of Figure 5).

Simulated data have also been used to evaluate the performance characteristics of the baseline design. Figures 6 and 7 show the effective area and modulation factor, respectively, as a function of incident photon energy. In both cases, are shown the results for two different detector depths $-5.08 \mathrm{~cm}$ (as depicted in Figure 4) and $7.62 \mathrm{~cm}$. Although the deeper detector clearly presents an advantage in terms of effective area, the varying detector depth appears to have little influence on the modulation factor. In practice, the advantage of increased effective area for a deeper detector must be offset by the decrease in light collection efficiency and the consequent effects on the detector threshold (Figure 11).

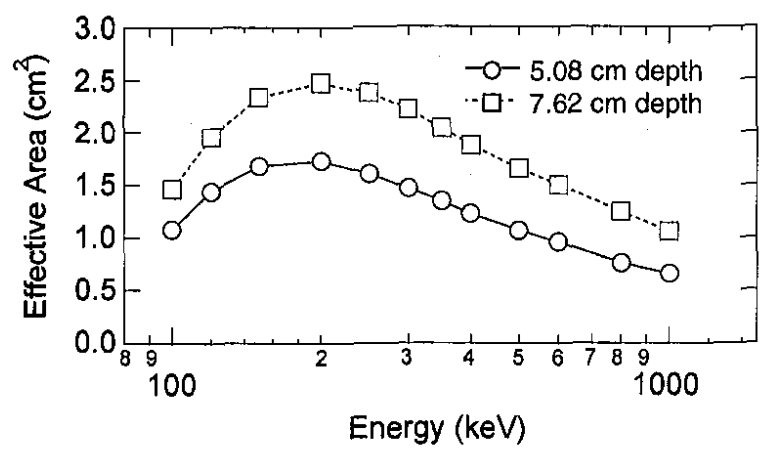

Figure 6: The effective area as a function of energy for the baseline design having a depth of both $5.08 \mathrm{~cm}$ and $7.62 \mathrm{~cm}$.

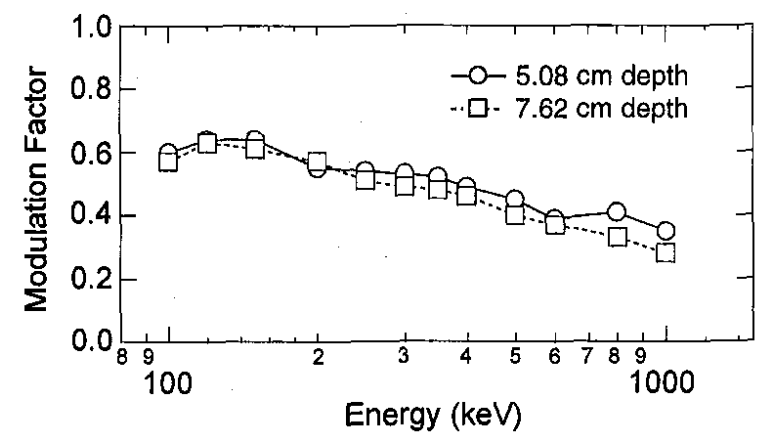

Figure 7: The modulation factor as a function of energy for the baseline design having depths of $5.08 \mathrm{~cm}$ and $7.62 \mathrm{~cm}$.

One potentially useful aspect of the SOLPOL design is that there exists a significant polarization response at large offaxis angles. This can be seen in Figure 8, which is based on simulations with a detector depth of $5.08 \mathrm{~cm}$. The effective area remains relatively constant at large angles. This results from the fact that the exposed geometric area of the detector remains relatively constant. Although there is a significant decrease in the modulation factor at large angles, there is still significant polarization response even at $60^{\circ}$ incidence angle. The off-axis response of this design would be very useful, for example, in studies of gamma-ray bursts. 


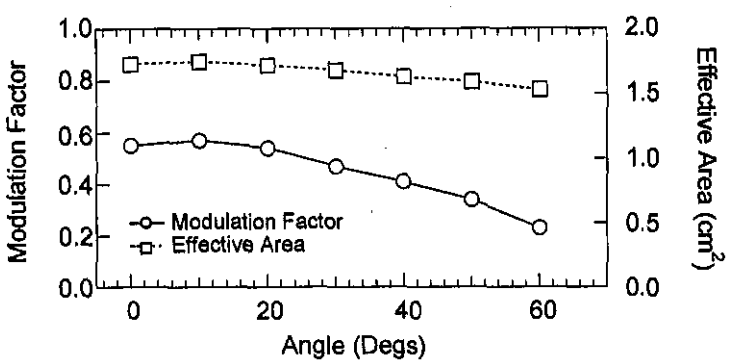

Figure 8: The modulation factor and effective area at $200 \mathrm{keV}$ for various incidence angles. The polarimeter maintains good response out to $60^{\circ}$ incidence angles.

\section{SCIENCE MODEL DEVELOPMENT}

Our recent work has concentrated on the fabrication of a science model based on the baseline SOLPOL design (Figure 4). Although the fabrication of the science model is not yet complete, we have made progress in several key technical areas.

\section{A. PSPMT Imaging Tests}

Our intial design incorporated the use of scintillating fibers as a scattering medium [6]. This choice was motivated by the fine (sub-mm) spatial resolution that could, in principle, be achieved. We have assembled and tested a PSPMT / fiberbundle module for the purpose of evaluating the imaging characteristics of such a device. The Bicron fiber bundle consisted of an $11 \times 11$ array of $3^{\prime \prime}$ long fibers, each with a cross-sectional area of $5 \times 5 \mathrm{~mm}^{2}$. The scintillating core of each fiber was based on BCF-10 scintillator. In addition to the standard PMMA cladding, each fiber was coated with an extramural absorber to reduce cross-talk between fibers. The fibers were viewed from one end by a $3^{N}$ square Hamamatsu R2487 PSPMT. Signal readout from the PSPMT was provided by a charge-divsion circuit. Readout of each event was triggered by a signal from the last dynode. The data processing and acquisition was achieved using a combination of NIM and CAMAC modules, with the final data recorded via a SCSI interface to a Power Macintosh computer running Kmax software.

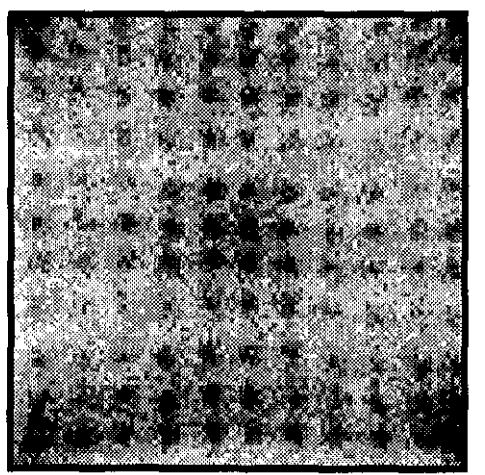

Figue 9: Fiber bundle flood test map based on uniform irradiance by $122 \mathrm{keV}$ photons from ${ }^{57} \mathrm{Co}$. The individual fiber elements (each $5 \times 5 \mathrm{~mm}^{2}$ ) can be clearly discerned.
Figure 9 shows the distribution of measured events resulting from a uniform irradiation of the front surface of the fiber bundle by $122 \mathrm{keV}$ photons from ${ }^{57} \mathrm{Co}$. The array of fibers is clearly defined. Also evident is the nonuniform nature of the PSPMT response.

The response of the PSPMT / fiber bundle module to a collimated beam of $662 \mathrm{keV}$ photons is shown in Figure 12 . The beam spot in this case was $\sim 3-4 \mathrm{~mm}$. The spatial response is dominated by a single fiber and its nearest neighbors. This suggests that individual events can be located with an accuracy comparable to the size of the plastic elements.
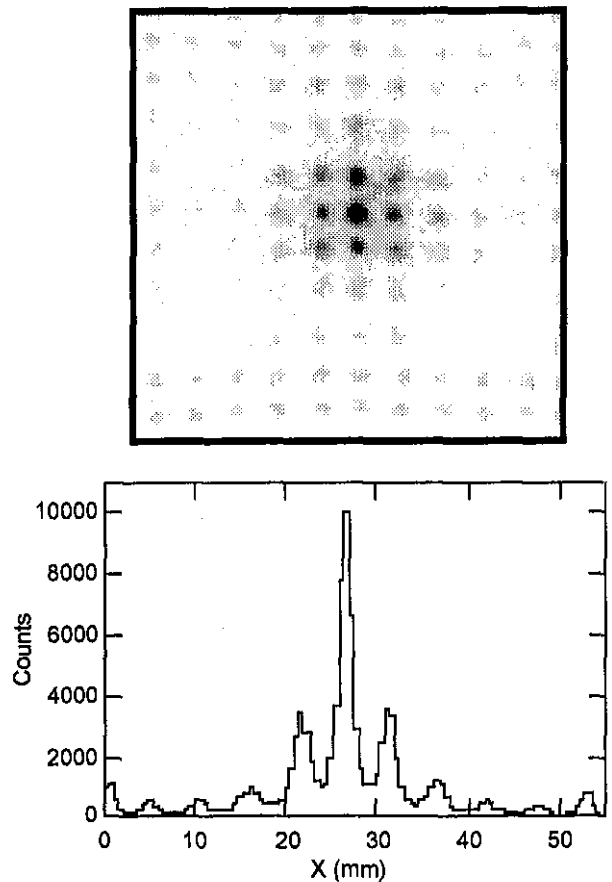

Figure 10: Distribution of events in the fiber bundle when irradiated with a collimated beam of $662 \mathrm{keV}$ photons from ${ }^{137} \mathrm{Cs}$. (Beam spot size $\sim 3-4 \mathrm{~mm}$.)

\section{B. Light Output of Scattering Elements}

The initial decision to use scintillating fibers led to a concern about the light collection efficiency and its potential impact on the energy threshold. Our ultimate goal is to achieve a polarimeter energy threshold of $50 \mathrm{keV}$. This requires a scattering element energy threshold of $15 \mathrm{keV}$. A major concern was whether such a low threshold energy could be achieved with scintillating fibers. Given the relatively large cross-sectional area that we were considering for the fibers, one potentially better alternative would be the use of individual plastic scintillating rods.

Motivated by these concerns, we made several laboratory measurements to determine the relative light output of scintillating fibers as compared to standard pieces of plastic scintillator. Specifically, we tested the light output of individual plastic scintillating rods with the same crosssectional area $\left(5 \times 5 \mathrm{~mm}^{2}\right)$ as our scintillating fibers, but of varying lengths $(2.54 \mathrm{~cm}, 5.08 \mathrm{~cm}$ and $7.62 \mathrm{~cm}$ ). The (Bicron BC-404) scintilator rods were individually wrapped in white plumbers tape to provide optical isolation and assembled into a 
$4 \times 4$ array. Tests were performed using a (non-imaging) $2^{\sim}$ $(5.08 \mathrm{~cm})$ PMT (EMI 9755NA).

The results of our testing with a ${ }^{133} \mathrm{Ba}$ source are shown in Figure 11. The relative light output of the various assemblies can be judged by the location of the Compton edge, which results primarily from $356 \mathrm{keV}$ photons interacting in the scintillator. These data show that the shorter geometries provide for greater light collection efficiency. More importantly, for the same (3") geometry, the light output of the individual scintillator rods is about a factor of 2.5 times the light output of the scintillating fibers. Although scintillating fibers might be preferred for very small cross sectional areas (ease of fabrication) or for very long geometries (light propagation), these results clearly argue in favor of using an array of individual plastic scintillator rods, rather than a scintillating fiber bundle.

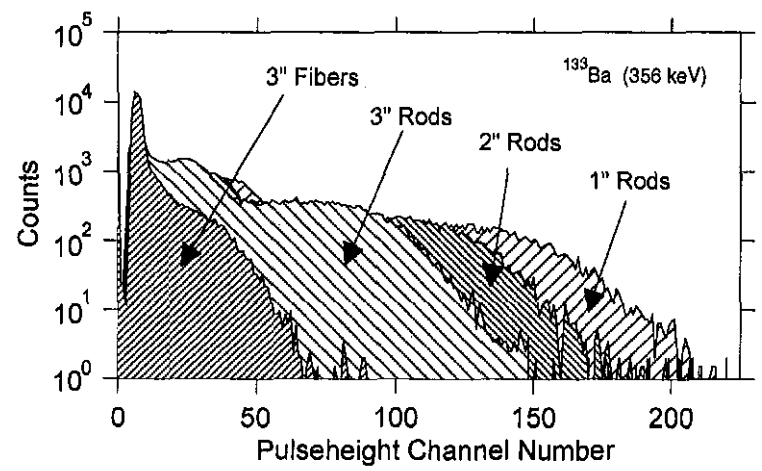

Figue 11: Spectra from ${ }^{133} \mathrm{Ba}$ recorded for different scintillator assemblies (of varying lengths) and for a scintillating fiber bundle. In all cases, the individual elements (or fibers) were $5 \times 5$ $\mathrm{mm}^{2}$. The relative light output of the scintillator assemblies is clearly superior to that of the scintillating fibers.

\section{Recent Progress}

We are presently working on the fabrication of the SOLPOL science model. The wrapping of 280 individual plastic scintillator elements has recently been completed. The final asembly and initial testing should take place early in 1999. Our initial imaging results using a bundle of these elements coupled to a 5" PSPMT are comparable to the results we achieved with the fiber bundle. The goals of these tests will be to evaluate the light output and spatial resolution of the scintillator array and to demonstrate the basic polarimetric capabilities of the device

For the initial science model fabrication, we have chosen a plastic element depth of 2 ". This will provide a reasonable level of light output, while retaining a large detection efficiency. (Further studies will be required to determine an optimum depth based on light output and detection efficiency considerations.) For the calorimeter elements, we will use an array of $1 \mathrm{~cm} \times 1 \mathrm{~cm}$ CsI elements cooupled to a Hamamatsu R5900-04 multi-anode PMT (MAPMT).

The initial tests will make use of the four readouts from a charge division network as supplied by Hammamatsu. This will provide a weighted average of the spatial distribution of the measured light output. Later tests will seek to make more effective use of the full spatial information afforded by the PSPMT using signals from the individual PSPMT anode wires. The R3292 PSPMT is designed with $28(\mathrm{X})$ plus $28(\mathrm{Y})$ cross-wire anodes. Rather than using all 56 individual channels, we plan to simplify the readout using only fourteen $(7 x, 7 y)$ anode wire sections. Other workers have succeeded in resolving individual $3 \mathrm{~mm}$ YAP crystal elements using such a readout- scheme and a center-of-gravity calculation for determining the interaction location [9]. The utility of this readout scheme for rejecting multiple scatter events will be investigated. If needed, we will more fully configure the PSPMT to test the multiple scatter event rejection at finer spatial scales. However, given the mean free path of photons in the plastic $(6 \mathrm{~cm}$ at $100 \mathrm{keV})$, we expect that a high level of multiple scatter event rejection can be achieved with the fourteen channel readout scheme.

In the future we may decide to explore alternative readout schemes. Despite the increased cost and complexity of having a large number of individual channels (one per detector element), the technical advantages may dictate such a course of development. Our science model testing will help us to evaluate the need for such alternative technologies

\section{SUMMARY}

The goal of these science model tests is to verify the performance characteristics of the SOLPOL design and to define the final electronics configuration. Once this has been accomplished, we can move forward with the detailed design and fabrication of a self-contained engineering model. We anticipate that this design would be used in the context of an array of polarimeter modules. For solar flares, we calculate that an array of 4 modules is capable of measuring sensitivity levels down to a few percent in X-class flares. A larger array of 16 modules would be capable of measuring solar flare polarization levels below $1 \%$ for the largest events and would also be capable of measuring polarization levels down to about $15 \%$ in some of the largest $\gamma$-ray bursts [5]. Although similar designs have been discussed in the literature $[10,11]$, we are unaware of any other active effort to specifically measure polarization in solar flares or in $\gamma$-ray bursts at energies above $100 \mathrm{keV}$.

In addition to its potential for studying transient sources, the SOLPOL design might also be useful in the context of an imaging polarimeter. For example, a SOLPOL element or array of elements could be used with a rotation modulation collimator to achieve arc-second angular resolution. Such an approach is not unlike that employed for hard X-ray imaging (without polarization capability) in the upcoming HESSI mission. The spatial information intrinsic to the SOLPOL design might also be useful in a coded-aperture system, although perhaps limited to arc-minute angular resolutions. We have recently embarked on an effort to evaluate the various possible imaging techniques that could be used with a SOLPOL-like device.

\section{ACKOWLEDGEMENT}

This work has been supported by NASA grants NAGW5704 and NAG5-7294. 


\section{REFERENCES}

[1] R.D. Evans, The Atomic Nucleus, New York: McGrawHill, 1958.

[2] R. Novick, "Stellar and solar X-ray polarimetry," Space Science Reviews, vol. 18, pp. 389-408, 1975.

[3] F. Lei, A.J. Dean and G.L. Hills, "Compton scatter polarimetry in gamma-ray astronomy," Space Science Reviews, vol. 82, pp. 309-388, 1997.

[4] M. McConnell, D. Forrest, K. Levenson, and W.T. Vestrand, "The design of a gamma-ray burst polarimeter," in AIP Conf. Proc. 280, Compton Gamma-Ray Observatory, M. Friedlander, N. Gehrels and D.J. Macomb, Eds. New York: AIP, 1993, pp. 1142-1146.

[5] M.L. McConnell, D.J. Forrest, J. Macri, J.M. Ryan, and W.T. Vestrand, "Development of a hard X-ray polarimeter for gamma-ray bursts," AIP Conf. Proc. 428, GammaRay Bursts, C.A. Meegan and P. Cushman, Eds. New York: AIP, 1998, pp. 889-893.

[6] M.L. McConnell, D.J. Forrest, J, Macri, M. McClish, M. Osgood, J.M. Ryan, W.T. Vestrand and C. Zanes
"Development of a hard X-ray polarimeter for solar flares and gamma-ray bursts," IEEE Trans. Nucl. Sci., vol. 45, no. 3, pp. 910-914, June, 1998.

[7] H. Sakurai, M. Noma, and H. Niizeki, "A hard x-ray polarimeter utilizing Compton scattering," in SPIE Conf. Proc., vol. 1343, pp.512-518, 1990.

[8] W.H. McMaster, "Matrix representation of polarization," Reviews of Mod. Phys., vol. 33, no. 1, pp. 8-28, January 1961.

[9] R. Wojcik, S. Majewski, B. Kross, D. Steinbach, and A.G., "High spatial resolution gamma imaging detector based on a $5^{\prime \prime}$ diameter R3292 Hamamatsu PSPMT," IEEE Trans. Nucl. Sci., vol. 45, no. 3, pp. 487-491, June, 1998.

[10] G. Chanan, A.G. Emslie, and R. Novick, "Prospects for solar flare X-ray polarimetry," Solar Physics, vol. 118, pp. 309-319, 1988.

[11] T.L. Cline, et al., "A gamma-ray burst polarimeter study," in Proceedings of the 25th Internat. Cosmic Ray Conf., vol. 5, pp. 25-28, 1997. 